\title{
A Case of Psychogenic Paraplegia Documented During Sleep by Video Monitoring
}

\author{
Hyung-Geun $\mathrm{Oh}^{1}$, Kyeong-Seok Lee ${ }^{2}$, Hyung-Kook Park ${ }^{1}$, Kwang-Ik Yang ${ }^{1}$ \\ Department of ${ }^{I}$ Neurology and ${ }^{2}$ Neurosurgery, College of Medicine, Soonchunhyang University, Cheonan Hospital
}

Psychogenic paraplegia is an uncommon conversion disorder characterized by nonorganic loss of motor function precipitated by some type of traumatic event. A 60-year-old man was referred from neurosurgery department with the chief complaint of paraplegia. One year ago he was involved in a motor vehicle accident. Whole spine magnetic resonance imaging (MRI) and electrophysiological study couldn't explain the organic cause of paraplegia. We decided to observe him during his sleep as we suspected a psychogenic cause for the symptoms. Irregular, non-stereotyped and involuntary leg movements were documented during sleep by video monitoring, and he was diagnosed as psychogenic paraplegia. In conclusion, if a psychogenic paraplegia is suspected, video monitoring during sleep could be recommended as a differentiation tool from neurological disorder.

Key Words : Psychogenic paraplegia, Video monitoring

\section{Introduction}

Neurologists are often confronted with patients presenting with neurological symptoms without any organic cause. The main feature of conversion disorder is the presence of symptoms or deficits that affect voluntary motor or sensory function, which suggest neurological or other general medical conditions. In general, the symptoms are not produced intentionally. ${ }^{1}$ Conversion disorder can present with motor symptom including weakness/paralysis of a limb or the entire body (psychogenic paralysis or motor conversion disorders).

According to the Diagnostic and Statistical Manual of Mental Disorders, $4^{\text {th }}$ edition, the following features are identified as positive criteria for the diagnosis of psychogenic paralysis: (1) contradictory clinical findings such as normal muscle tone, normal deep reflexes, or pyramidal signs; (2) paradoxical behavior; (3) normal electrophysiological features; and (4) normal radiologic findings. ${ }^{1}$ Conversion often

\footnotetext{
* Address of correspondence Kwang-Ik Yang Department of Neurology, College of Medicine, Soonchunhyang University, Cheonan Hospital 23-20 Bongmyeong-dong, Cheonan-si, Chungnam, 330-721, Korea

Tel: +82-41-570-2290 Fax: +82-41-579-9021

E-mail: kiyang@sch.ac.kr
}

follows a traumatic event and may be caused by a psychological mechanism evoked to cope with acute stress. ${ }^{2}$ Physicians are faced with a situation in which patient's symptoms are not completely compatible with the description of the original accident or the physical examination. ${ }^{3-6}$ However, if presence of leg movements could be documented during sleep in patient with psychogenic paraplegia, physicians may differentiate it from neurological disorder easily.

The present report describes the utility of video monitoring during sleep to differentiate psychogenic paraplegia from neurological disorder.

\section{Case report}

A 60-year-old man was referred from neurosurgery department with the complaint of paraplegia. One year ago he had been involved in a motor vehicle accident. He had multiple facial abrasions, cerebral concussion, nasal bone fracture, left lateral malleolar synovitis, and delirium. On arrival at the emergency room of the local hospital, he had been noted to be paraplegic with normal rectal tone. Otherwise, he had looked apparently healthy with stable vital signs. He had been admitted in that hospital for 6 months. Despite inten- 


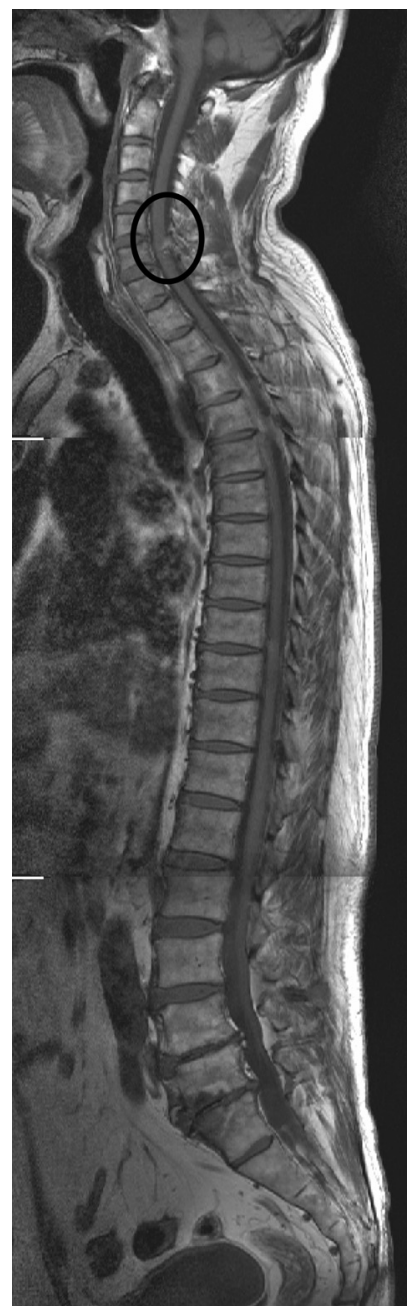

Figure 1. MoThe MRI of the whole spine showed mild lumbar spinal stenosis and ossification of ligamentum flavum at right $\mathrm{C} 6$ level in the circle. But the MRI failed to show any evidence of spinal cord, conus medullaris, or cauda equina compression.

sive physical therapy, he had not recovered from paraplegia. Repeated spine MRIs had not showed any abnormal findings as an organic cause for the paraplegia.

He was referred to neurosurgery department in our hospital for independent medical examination. A detailed neurological examination demonstrated an alert and oriented patient who was evasive when answering questions. His upper extremities had normal sensation and motor function. His lower extremities had complete loss of motor and sensory functions and no sensory level. He had normal reflexes in the upper and lower extremities. There was no specific pathologic reflex. His lumbar and thoracic spines were nontender without deformities and he had normal rectal sphincter tone/bladder function and a normal bulbo-cavernosus reflex. His whole spine MRI showed mild lumbar spinal stenosis and ossification of ligamentum flavum at right C6 level (Figure 1). But the MRI failed to show any compression lesions of spinal cord, conus medullaris and cauda equina. The patient had also a brain CT imaging, which was normal. Somatosensory evoked potential (SSEP) study didn't show any definite abnormal finding. A psychiatric consultation was sought to evaluate this patient for a possible conversion reaction. The psychiatrist couldn't conclude any psychogenic cause of the paraplegia from his interview. So we observed him during sleep with video monitoring and monitored the patient for about 7 hours overnight by video. We looked at irregular, frequent, non-stereotyped and spontaneous both leg movements during sleep. So we considered his paraplegia to be psychogenic. After this study, the patient was lost to followed-up.

\section{Discussion}

A diagnosis of conversion disorder should be made only after a thorough medical investigation has been performed to rule out neurological or general medical conditions. ${ }^{1}$ Conversion symptoms are often inconsistent. A 'paralyzed' extremity will be moved inadvertently while getting dressed, or when attention is directed elsewhere. A broad range of neurological conditions may be misdiagnosed as conversion disorder.

Psychogenic paraplegia after trauma presents a difficult medicolegal issue. Despite normal physical examination, studies are often necessary to exclude lesions such as disc herniation, epidural hematoma, or contusion of the spinal cord. ${ }^{6}$ The expected objective signs such as reflex changes following paraplegia are rarely present. So the diagnosis of nonorganic paraplegia is not easy.

SSEP or motor evoked potential (MEP) recording are simple and noninvasive methods which can objectively evaluate the integrity of central and peripheral sensory and motor pathways. ${ }^{7-8}$ The use of SSEP has expanded to the evaluation of trauma, stroke, tumors, and metabolic disor- 
ders. $^{7}$ In some cases, normal SSEP or MEP in a patient denying sensation of the stimulus offer objective evidence of the psychogenic nature of the paraplegia and are a valuable tool in this clinical setting. There was a report that PSG can be helpful in confirming the psychogenic nature of the neurologic deficit. ${ }^{9}$

Lee et al reported abnormal lower limb movements during sleep in three individuals with spinal cord injury at the thoracic level and related them to periodic limb movements (PLM). ${ }^{10}$ PLM is characterized by periodic episodes of repetitive, highly stereotyped, limb movements that occurs during sleep. It typically involves extension of the big toe, often in combination with partial flexion of the ankle, the knee, and sometimes the hip. These movements, when they occur, are repeated in a regular manner at 5 to 90 seconds intervals, with each movement lasting 0.5 to 5.0 seconds. Leg movements of our patient could be distinguished from PLMs by their non-stereotypic, non-periodic occurrence

Because we did not perform PSG including EEG and surface EMG but only video monitoring, we had limitation to judge sleep or wake state during this study. Although we were inconvenienced by not doing PSG study, irregular, non-stereotyped and spontaneous leg movements were different from PLM by video monitoring. So we diagnosed him as psychogenic paraplegia. Therefore we suggest that video monitoring during sleep may be a useful tool for evaluating the psychogenic nature of the patient with paraplegia.

\section{REFERENCES}

1. American Psychiatric Association. Conversion Disorders. In: Diagnostic and Statistical Manual of Mental Disorders. $4^{\text {th }}$ ed. Washington DC: American Psychiatry Association. 1994.

2. Ford CV. Somatoform disorders. In: Ebert MH. Current Diagnosis and Treatment in Psychiatry. $1^{\text {st }}$ ed. McGraw-Hill: New York, 2000, pp.366-377.

3. Shell MG, Mitchell HL, Loes MW, Belan AP. Conversion disorder presenting in a patient with an implantable morphine pump and an epidural abscess resulting in paraplegia. Arch Phys Med Rehabil 1997;78:226-229.

4. Berhane L, Kurman R, Smith S. Lower extremity paralysis after operative laparoscopy from conversion disorder. A case report. J Reprod Med 1998;43:831-835.

5. Crimlisk HL, Bhatia K, Cope H, David A, Marsden CD, Ron MA. Slater revisited: 6 year follow up study of patients with medically unexplained motor symptoms. BMJ 1998;21:582-586.

6. Laraki M, Orliaquet GA, Flandin C, Merckx J, Barrier G. Hysterical paraplegia as a cause of transient paraplegia after epidural anesthesia. Anesth Analg 1996;83:876-877.

7. Haghighi SS, Meyer S. Psychogenic paraplegia in a patient with normal electrophysiologic findings. Spinal Cord 2001;39:664-667.

8. Cantello R, Boccagni C, Comi C, Civardi C, Monaco F. Diagnosis of psychogenic paralysis: the role of motor evoked potentials. $J$ Neurol 2001;248:889-897.

9. Jahn K, Arnold S, Zingler VC, Strupp M, Brandt T. Polysomnographic as a therapeutic aid in of psychogenic functioning paraplegia. Nervenarzt 2006;77:948-951. Abstract.

10. De Mello MT, Silva AC, Esteves AM, Tufik S. Reduction of periodic leg movement in individuals with paraplegia following aerobic physical exercise. Spinal Cord 2002;40:646-649. 